\title{
THE EFFICACY OF SURGICAL TECHNIQUES FOR CERVICAL SPONDYLOTIC MYELOPATHY ON FUNCTIONAL OUTCOME, RECOVERY AND PATIENT SATISFACTION
}

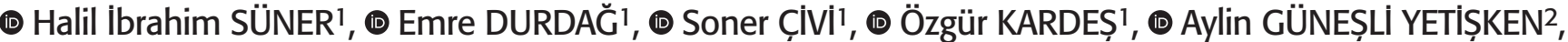 \\ (1) Kadir TUFAN ${ }^{1}$, (1) Metin ÖZALAY3
}

\author{
${ }^{1}$ Baskent University, Adana Dr. Turgut Noyan Application and Research Center, Department of Neurosurgery, Adana, Turkey \\ ${ }^{2}$ Baskent University, Adana Dr. Turgut Noyan Application and Research Center, Department of Radiology, Adana, Turkey \\ ${ }^{3}$ Ortogrup Clinic, Department of Orthopaedics and Traumatology, Adana, Turkey
}

\begin{abstract}
Objective: Cervical spondylotic myelopathy is a neurological condition that develops due to degenerative changes of the spine resulting in compression of the spinal cord and its vascular structures. It is the most common form of spinal cord injuries in adults; however, its diagnosis is often delayed due to the insidious progression of the disease. We investigated the functional outcome, recovery and satisfaction of patients undergoing surgical treatment in our clinic.

Materials and Methods: The study included patients who were operated for spinal stenosis caused by cervical spondylosis. The patient's age, sex, admission complaint, duration of complaint, comorbidities, neurological examinations and gait performances were evaluated, measured by the Nurick Scale. The spinal cord was examined with the preoperative Magnetic Resonance imaging. The operative times, surgical techniques, complications, post-discharge follow-up durations, functional outcomes and long-term complaints of the patients were analysed. We divided the patients into four groups according to the surgical technique.

Results: A total of 50 patients were operated, of which 38 were males and 12 were females $(\mathrm{M} / \mathrm{F}=3.16)$, with a mean age of 68.8 years. Of these patients, 24 (48\%) underwent only laminectomy (group 1), six (12\%) underwent laminectomy and fusion (group 2), 17 (34\%) underwent corpectomy and anterior fusion (group 3) and three (6\%) underwent combined surgery (group 4). Recovery was seen in $60 \%$ of all the patients, while $6 \%$ had deterioration in myelopathy. The patients in group 1 had the shortest operative time and length of hospital stay, while those in group 2 had the highest satisfaction and recovery rate (83.33\%).

Conclusion: Age, duration of symptoms and neurological condition at admission are the most important determinants of the response to treatment. Patients who are clinically and radiologically diagnosed should be treated with surgery as soon as possible, and restoration of cervical alignment, decompression and if necessary, fusion should be effectively performed in the surgery.

Keywords: Cervical spondylotic myelopathy, corpectomy, laminectomy, stenosis, spinal cord
\end{abstract}

\section{INTRODUCTION}

Cervical spondylotic myelopathy (CSM) is a poor neurological condition that develops due to degenerative changes of the spine resulting in compression of the nearby spinal cord over time. The most common cause of spinal cord dysfunction in adults worldwide is $\operatorname{CSM}^{(1,2)}$, and it typically presents with decreased hand skills, gait and balance difficulties caused by dysfunction in fine motor movements. In the progression of the disease, slow, gradually increasing upper and lower extremity sensorimotor dysfunction and sphincter dysfunction occur; yet, in very few cases, rapid neurological deterioration may occur ${ }^{(3)}$. The incidence of CSM is likely to increase with increasing age in accordance with its degenerative aetiology.
It is well known that in CSM, an effective treatment option is surgical decompression of the spinal cord, as it does not only halts the progression of symptoms, but also shows a significant functional improvement in a considerable proportion of individuals treated ${ }^{(4,5)}$. Pathologies located in the anterior or posterior spinal canal can be the cause of spondylotic spinal compression and accordingly, surgical decompression can be performed using an anterior or posterior surgical approach. Anterior surgery is typically performed in the form of anterior cervical discectomy and fusion or corpectomy and fusion. Posterior surgery refers to laminoplasty or laminectomy with or without fusion ${ }^{(3)}$.

Although it is generally safe and effective, $11-38 \%$ of CSM patients treated surgically develop complications ${ }^{(6,7)}$. These include dysphagia, $\mathrm{C} 5$ radiculopathy, wound infection, axial pain 
and postoperative (post-op) kyphosis ${ }^{(8)}$. Today, it remains unclear as to whether multi-level spondylotic cervical spinal stenosis can best be treated with the anterior or posterior surgical approach and whether each of these surgical approaches is superior in terms of patient outcomes or complication rates. The aim of this study is to investigate the effects of the surgical techniques used in patients with CSM operated in our clinic on complication, recovery and patient satisfaction.

\section{MATERIALS AND METHODS}

Our study included only patients who were operated for spinal stenosis caused by cervical spondylosis. The exclusion criteria in our study involved patients with spinal stenosis who were operated for trauma, tumour and other aetiologies. The patients' age, sex, admission complaint, duration of complaints, systemic diseases and neurological examinations were evaluated. Their gait performances were evaluated according to the Nurick scale. Stenosis levels and the presence of myelomalacia were examined with the preoperative (preop) magnetic resonance imaging (MRI) examinations of the cervical vertebra. The operative times, surgical approaches, number of decompressions, peroperative (per-op) and post-op complications, post-discharge follow-up durations, functional outcomes and long-term complaints of the patients were analysed. In addition, the post-op length of hospital stay, complications, functional outcomes and long-term complaints of the patients were analysed according to surgical approaches. The patients were divided into four groups according to the surgical technique: group 1 (laminectomy without fusion), group 2 (laminectomy and fusion), group 3 (anterior corpectomy and fusion) and group 4 (combined surgery).

This study was approved by Başkent University Medicine and Health Sciences Research Committee (94603339604.01.02/845).

\section{Selection of the Surgical Technique}

When CSM is diagnosed, the type of treatment is discussed. The treatment of CSM should be with surgery or conservatively. CSM is generally considered a surgical disease, because symptoms tend to worsen in natural course. Therefore, in our clinic, all cases of CSM with clinical and radiological consistency were treated with surgery.
The surgical treatment of CSM is performed by anterior, posterior or combined approach, depending on the specific pathology. The patients with 1 to 2 vertebral level kyphosis or ossification of the posterior longitudinal ligament were generally operated with an anterior approach. The patients with $>3$ levels of cervical stenosis, posterior compression or congenital stenosis, laminectomy and posterior fusion were generally performed.

\section{Statistical Analysis}

Statistical analysis was performed using the statistical package SPSS software (Version 17.0, SPSS Inc., Chicago, IL, USA). If the continuous variables were normal, they were described as the mean \pm standard deviation $[p>0.05$ in Shapira-Wilk $(n<30)]$, and if not normal, they were described as the median. Comparisons between groups were applied using Kruskal-Wallis test, used for data not normally distrubited. The chi-square test or Fisher's exact test. Test was used to analyse the catagorical variables between the groups. Values of $p<0.05$ were considered statistically.

\section{RESULTS}

A total of 50 patients were operated in our hospital between 2014 and 2019 of which 38 were male and 12 were female $(M / F=3.16)$. The mean age was 68.8 years $(43-85)$. The most common admission complaints were simultaneous weakness in the arms and legs, difficulty in walking, arm pain, weakness only in the legs, weakness only in the arms, weakness on one side of the body and spasticity. The mean time from onset of symptoms to presentation was 8.8 months ( 2 days-60 months). The patients' personal history evaluated revealed that the most common systemic disease was diabetes mellitus (DM) with $34 \%$ of the patients $(n=17)$, followed by coronary artery disease $(n=15)$, and hypertension ( $n=15)$.

The neurological examinations of the patients revealed that 35 patients (70\%) had pathological reflex (Hofmann, Clonus and Babinsky), while 23 (46\%) patients had quadriparesis, eight (16\%) patients had paraparesis, eight (16\%) patients had monoparesis, four patients (8\%) had hemiparesis and two patients (4\%) had spastic paraparesis. Of the patients, $10 \%$ were grade $0,16 \%$ were grade $1,8 \%$ were grade 2 and $66 \%$ were grade 3 and 4 according to the Nurick scale. None of the patients was grade 5 (Table 1). During the outpatient clinic

Table 1. Our patients were evaluated according to the Nurick scale (Evaluation 0 to 5 Points) preoperatively. Of the patients, $10 \%$ were grade $0,16 \%$ were grade $1,8 \%$ were grade 2 and $66 \%$ were grade 3 and 4 according to the Nurick scale. None of the patients was grade 5

\begin{tabular}{lll} 
Grade & Description & Our cases \\
\hline 0 & Signs and symptoms of root involvement without spinal cord disease & $10 \%$ \\
\hline 1 & Signs of spinal cord disease without difficulty in walking & $16 \%$ \\
\hline 2 & Slight difficulty in walking that does not prevent full-time employment & $8 \%$ \\
\hline 3 & $\begin{array}{l}\text { Difficulty in walking that prevents full-time employment or daily life without } \\
\text { requiring assistance with walking }\end{array}$ & $66 \%$ \\
\hline 4 & Ability to walk only with assistance & - \\
\hline 5 & Chair bound or bedbound &
\end{tabular}


admission of the patients, the MRI of the cervical vertebra performed showed that 32 (64\%) patients had myelomalacia of the spinal cord. Of the patients, 25 (50\%) had spinal stenosis at 2 levels, $20(40 \%)$ at 3 levels and $5(10 \%)$ at 1 level. Spinal stenosis was most commonly observed at C4-5 (80\%), followed by C5-6 (64\%), C3-4 (48\%) and C6-7 (34\%).

Of the patients, 24 (48\%) underwent laminectomy without fusion (group 1), six (12\%) underwent laminectomy and fusion (group 2), 17 (34\%) underwent corpectomy and anterior fusion (group $3)$, three (6\%) underwent anterior and posterior decompression and fusion (group 4) (Figure 1). Decompression was performed

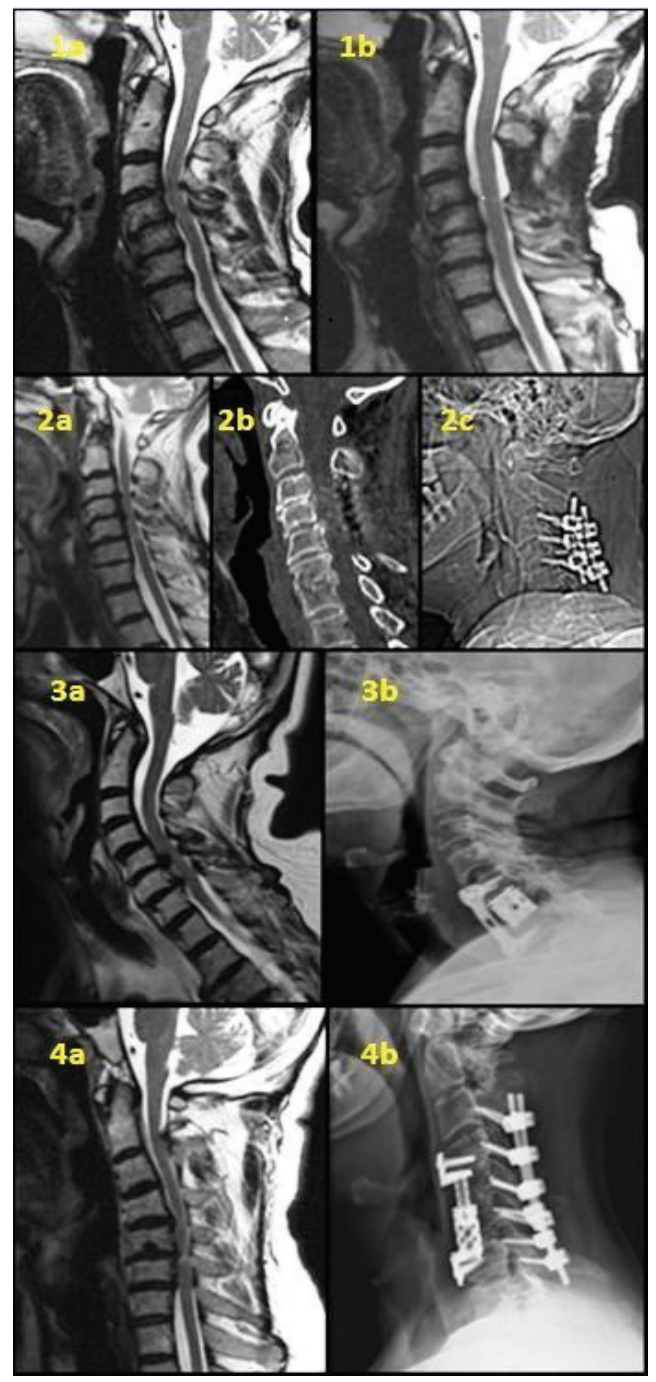

Figure 1. Case samples from groups; $\mathbf{1 a}$, b: Preoperative and postoperative MRI images of one patient who underwent laminectomy (group 1), 2a, b, c: Preoperative MRI and postoperative CT control of a patient who underwent laminectomy and posterior fusion (group 2), 3a, b: Preoperative MRI and postoperative X-ray control of a patient who underwent anterior corpectomy and fusion (group 3), 4a, b: Preoperative MRI and postoperative X-ray control of a patient who underwent anterior and posterior decompression and fusion (group 4)

MRI: Magnetic resonance imaging, CT: Computed tomography at 2 levels in 19 patients, 1 level in 17 patients and 3 levels in 14 patients. The mean operative time of the surgical groups was examined in minutes (min). The mean operative time was 102.5 minutes in group 1, 210 minutes in group 2,175.2 minutes in group 3 and 220 minutes in group 4 (Table 2). There was a statistically significant difference $(p=0.001)$ in the mean operative time of the surgical groups. In addition, the shortest post-op length of hospital-stay (3.66 days) was found in group 1 , although this was not statistically significant $(p=0.572)$. Considering the per-op and post-op complications of all the patients, this rate was $10 \%$. Two of the patients who underwent only laminectomy were re-operated due to spinal cord oedema and haematoma at the operation site after post-op 24 hours, and two of the patients who underwent corpectomy and anterior instrumentation were re-operated due to hematoma at the operation site (post-op $2^{\text {nd }}$ day) and corpectomy cage shift (post-op $4^{\text {th }}$ day). Moreover, one of the patients who underwent laminectomy and fusion peroperatively developed dural tear, which was repaired in the same session (Table 2). Complication rates were not statistically significant $(p=0.978)$.

The mean post-discharge follow-up period of the patients was 12.82 (1-48 months) months. The post-discharge followup analysis of all the patients with CSM revealed that, of the patients, 30 (60\%) achieved complete recovery, nine (18\%) achieved partial recovery and 5 (10\%) got worse compared to the preoperative period (increase in motor loss in three patients, spasticity in two cases), while six (12\%) had no change compared to the pre-op period. The complete recovery rates of the surgery groups were as follows, $83.33 \%$ of group 2 and $70.58 \%$ of group 3 showed complete recovery, while this rate in group 1 was $41.1 \%$ (Table 2). There was no significant difference of recovery rates between surgical groups in this context ( $p=0.657)$. One patient died of acute coronary syndrome (ACS) 7 days after discharge. We determined that nine (18\%) patients developed neuropathic pain complaints in our longterm follow-up, more than half of whom (five patients) were in the group treated with only laminectomy, and appropriate medical treatment was given to these patients.

\section{DISCUSSION}

The most common cause of spinal cord dysfunction in individuals older than 55 years is $\operatorname{CSM}^{(9)}$. Cervical spondylosis is a progressive disease characterised by degenerative changes affecting the vertebrae, intervertebral discs, facets and associated ligaments. These changes accelerate CSM by causing narrowing of the canal vertebralis diameter and direct compression of the spinal cord and/or surrounding blood vessels ${ }^{(10)}$. Disruption in blood supply to the spinal cord tissue, further increasing neuronal injury is caused by the vascular involvement. The disease can result in long-term disability and severe neurological disorders. Early and effective treatment before irreversible spinal cord injury develops is important to maintain the quality of life of these patients. 
Table 2. Operative times, complications, post-op length of hospital stay and treatment outcomes of patients by surgeries

$\begin{array}{lllll}\begin{array}{l}\text { Number of } \\ \text { patients }\end{array} & \begin{array}{l}\text { Operative time } \\ (\mathrm{min})\end{array} & \begin{array}{l}\text { Complication } \\ (\mathrm{n})\end{array} & \begin{array}{l}\text { Length of } \\ \text { stay (day) }\end{array} & \begin{array}{l}\text { Outcome } \\ (\%)\end{array} \\ & (p=0.001) & (p=0.978) & (p=0.572) & (p=0.657)\end{array}$

\begin{tabular}{|c|c|c|c|c|c|}
\hline Group 1 & 24 & 102.5 & 2 & 3.66 & $\begin{array}{l}\text { Complete recovery: } 41.66 \\
\text { Partial recovery: } 25 \\
\text { No change: } 20.83 \\
\text { Worsening: } 12.5\end{array}$ \\
\hline Group 4 & 3 & 220 & - & 6.33 & $\begin{array}{l}\text { Complete recovery: } 66.66 \\
\text { Partial recovery: } 33.33 \\
\text { No change:- } \\
\text { Worsening:- }\end{array}$ \\
\hline
\end{tabular}

post-op: Postoperative

The progression of cervical myelopathy is often insidious although it is seen only in a small portion of patients with spondylosis. The natural course of CSM is variable. Some patients show a gradual worsening, while others have a long silent period. Minor and major traumas that may occur in the presence of cervical spondylosis can cause acute clinical deterioration and central cord syndrome. The symptoms of some of the patients in this study had started within 1 month, and their condition had worsened within days.

Patients usually present gait disturbance and fine motor dysfunction since the spinocerebellar and corticospinal pathways are affected in the first place ${ }^{(11)}$. Therefore, patients exhibit hand numbness and hand motor dysfunctions, a widebased and ataxic gait and inability to perform tandem standing during the initial assessment. Neurological examination shows lower motor neuron findings at the highest stenosis level and upper motor neuron findings at lower levels. Positive Hoffman, Clonus and Babinski reflexes and motor weakness are frequently encountered ${ }^{(12)}$. In this study, the most common admission complaints of the patients were weakness in the arms and legs and walking difficulties, while pathological reflexes such as motor weakness with $78 \%$, Hoffman, Clonus and Babinski reflexes with $70 \%$ were observed.

Nurick ${ }^{(13)}$ in 1972 published the original symptom severity scale for CSM and was based only on gait disturbance. In recent years, this scale has been considerably replaced by a more holistic rating system, called the Japanese Orthopaedic Association Myelopathy Evaluation Questionnaire (JOA scale) (14). The Nurick scale is still very much in use to assess the effect of gait dysfunction on daily life activities however, the JOA scale has become the preferred rating scale to assess overall patient weakness. We found out using the Nurick scale that, $66 \%$ of the patients were grade 3 and 4 in the pre-op period.
Neurological changes developing with DM cause axonal damage in the spinal cord. Sensory findings usually include proprioceptive loss and loss of glove-like sensations in the hands that can be confused with DM or concomitant peripheral neuropathy ${ }^{(10)}$. The JOA scale scores of those with DM were lower than those of other patients was the discovery of a study evaluating patients with CSM who were recently treated surgically ${ }^{(15)}$. In our study, $34 \%$ of the patients had DM. Nine of the patients had neuropathic pain in post-op course and three of these patients had DM previously. Complaints such as neuropathic pain and sensory loss can be of DM origin Electromyography (EMG) can be used to confirm this, but some patients in our study did not have EMG examination.

A very important method to confirm the diagnosis of CSM by imaging. Plain radiographs, computed tomography (CT) and MRI with or without myelography can be used to evaluate spinal canal narrowing and pathological vertebral changes. Plain radiographs are usually taken before advanced modalities because they are cheaper, faster and expose the patient to less radiation. However, due to the non-invasive nature, high resolution and ability to show soft tissues in detail of MRI, it is preferred for precise evaluation ${ }^{(16)}$. Sometimes, an increased T2 signal is visualised in the spinal cord on MRI. This condition, which we call myelomalacia, suggests spinal cord injury and permanent damage due to spinal cord compression or recurrent trauma ${ }^{(12)}$. In our study, pre-op MRI examination was performed in all the patients, and myelomalacia was visualised in $64 \%$ of the patients. Pre-op CT examination was also performed in patients who were considered to have posterior longitudinal ligament ossification (PLLO), osteophyte formation and facet hypertrophy to evaluate these patients.

The most important risk factors for disease progression and worsening are age and duration of symptoms ${ }^{(10,17)}$. In addition, 
preoperative neurological function along with these factors are the most important prognostic indicators of surgical treatment success. The best treatment option should be decided taking into account these factors ${ }^{(12)}$. Indeed, the acceptable mean duration of symptoms ( 8.8 months) and mean age (68.8 years) of the patients we operated may be the cause of the good neurological condition at discharge and during the follow-up of the patients. In severe cases of CSM, surgery is often considered the best treatment option. Some studies have shown that 23$54 \%$ of patients who are initially treated with conservative treatment before surgery are later treated surgically ${ }^{(18)}$.

The most accurate surgical approach is not always clear. The goals of surgery for patients with CSM are decompression of the spinal cord, restoration of the cervical alignment and treatment of the instability, if any ${ }^{(19)}$. An AO Spine North America CSM study showed that cervical decompression halted worsening in patients regardless of the disease severity and the improved neurological outcomes, functional status and quality of life ${ }^{(20)}$. The anterior approach is preferred when the number of affected levels is 1 or 2. Discectomy and fusion or corpectomy and fusion can be included in the procedures performed during the anterior surgical approach. The anterior approach has been preferred by many spinal surgeons in recent years due to its advantages such as direct decompression of pathologies located in the anterior cervical spine (osteophyte, PLLO, disc herniations), the ability to resolve radiculopathy, muscle-preserving dissection to minimise post-op pain, low infection rates and correction of cervical kyphosis ${ }^{(10)}$.

The risk of complications of the anterior approach increases in the case of three or more levels and thus, the posterior approach should be considered in such cases. However, the posterior approach should not be used in the case of kyphosis. The extension of the spinal cord along the kyphotic spine causes neural injury, which can be exacerbated by posterior decompression. In our study, we found that combinations with the posterior approach were performed on all the patients with three levels of spinal stenosis, and the anterior approach alone was not performed on any of them. In the past, laminectomy without fusion was widely used for the treatment of CSM; however, due to the identification of post-laminectomy kyphotic deformities, the use of this technique has reduced ${ }^{(3)}$. Therefore, although the idea of adding fusion to the posterior approach has gained importance, restricted cervical mobility, neck stiffness and adjacent segment degeneration are its important handicaps ${ }^{(9)}$. In our study, we found that $48 \%$ of the CSM patients underwent laminectomy without fusion; this surgical approach was preferred more in high-risk patients due to advanced age and systemic diseases, and complaints such as neuropathic pain in the post-op long-term follow-up were most commonly observed in these patients. Although no postlaminectomy kyphotic deformity was observed in the follow-up of any patient, this group had the shortest operative time and post-op length of hospital-stay of 102.5 minutes and 3.66 days, respectively.
In our study, we found that the group in which only the anterior approach was preferred had one of the highest satisfaction rates $(70.58 \%)$, and only 1 or 2 levels of corpectomy were performed in this group. However, it was noted that $5.88 \%$ of the patients in this group and $12.5 \%$ of the patients who underwent laminectomy without fusion had post-op worsening. In addition, although the number of patients $(n=3)$ in the group in which the anterior and posterior approach was combined was small, the satisfaction rate of these patients (66.66\%) was better than that of the group (41.66\%) who underwent laminectomy without fusion. However, the patients treated with the combined approach had the longest length of hospital-stay. The group treated with laminectomy and fusion was found to be the best in terms of patient satisfaction and functional recovery $(83.33 \%)$.

Lawrence et al.(3) reviewed five studies and compared the success of their CSM surgical techniques. They found that a better functional improvement was observed after the anterior surgical treatment in two studies. More success was achieved after the posterior surgery approach in two studies. In one study, no difference was found between the anterior and posterior approaches. Only one of them was statistically significant. Thus, in the current literature, the anterior and posterior neurological outcome is insufficient for explaining the best surgical approach. We found that laminectomy and fusion was the most successful surgical method. However, the anterior approach is also a successful treatment option.

In the literature, post-op early and late complication rates have been reported as $15.6 \%$ and $4.4 \%$, respectively ${ }^{(20)}$. The more common complications are cardiopulmonary problems (3.3\%), dysphagia (3.0\%), superficial infection (2.3\%), pseudoarthrosis (1.8\%), C5 radiculopathy/palsy (1.7\%), worsening myelopathy (1.3\%), epidural/wound hematoma (1.0\%) and dural tear (1.0\%). Wound infection is more common in the posterior approaches ( $4.7 \%$ posterior, $0.6 \%$ anterior), while C5 radiculopathy/palsy is equally common in both approaches $(1.9 \%$ posterior, $1.7 \%$ anterior), and dysphagia is slightly more common in the anterior approach $(0.9 \%)^{(16,20)}$. The complication rate of all the patients in our study was $10 \%$. The rate of worsening myelopathy was $6 \%$, followed by hematoma at the operation site with $4 \%$, and per-op dural tear with $2 \%$. One patient who underwent laminectomy and fusion and recovered completely died of ACS approximately one week after discharge. Our complication rates were different compared to those of larger studies, since our sample size was small.

\section{CONCLUSION}

CSM is a degenerative disease that can be easily overlooked by clinicians, often leading to a delay in diagnosis and an irreversible spinal cord injury. Therefore, it should be treated as soon as possible. Laminectomy without fusion has the advantages of having the shortest length of hospital-stay and operative time. However, we recommend that laminectomy without fusion only 
be performed on high-risk patients due to comorbidities with 1 or 2 level involvement and no kyphosis, since it has more side effects such as neuropathic pain, lower functional recovery and patient satisfaction rates compared to other approaches. It will be useful to add fusion to multi-level laminectomies. We are of the opinion that laminectomy and fusion may be more successful in eligible cases in terms of patient satisfaction and functional recovery, and may cause fewer complications. When deciding on the surgical technique, it will be best to make a decision by evaluating the patient's age, clinical condition and radiological characteristics all together. Yet, there is a need for series with a larger sample size.

\section{Ethics}

Ethics Committee Approval: This study was approved by Başkent University Medicine and Health Sciences Research Committee (94603339-604.01.02/845).

Informed Consent: Informed consent was obtained from all patients.

Peer-review: Externally and internally peer-reviewed.

\section{Authorship Contributions}

Concept: E.D., Design: K.T., M.Ö., Data Collection or Processing: Ö.K., A.G.Y., Analysis or Interpretation: H.İ.S., S.Ç., Literature Search: H.I.S., S.Ç., A.G.Y., Writing: H.I.S.

Conflict of Interest: No conflict of interest was declared by the authors.

Financial Disclosure: The authors declared that this study received no financial support.

\section{REFERENCES}

1. Kalsi-Ryan S, Karadimas SK, Fehlings MG. Cervical spondylotic myelopathy: the clinical phenomenon and the current pathobiology of an increasingly prevalent and devastating disorder. Neuroscientist. 2013;19:409-21.

2. Tracy JA, Bartleson JD. Cervical spondylotic myelopathy. Neurologist. 2010;16:176-87.

3. Lawrence BD, Jacobs WB, Norvell DC, Hermsmeyer JT, Chapman $\mathrm{JR}$, Brodke DS. Anterior versus posterior approach for treatment of cervical spondylotic myelopathy: a systematic review. Spine (Phila Pa 1976). 2013;38(22 Suppl 1):173-82.

4. Furlan JC, Kalsi-Ryan S, Kailaya-Vasan A, Massicotte EM, Fehlings MG. Functional and clinical outcomes following surgical treatment in patients with cervical spondylotic myelopathy: a prospective study of 81 cases. J Neurosurg Spine. 2011;14:348-55.
5. Sampath P, Bendebba M, Davis JD, Ducker TB. Outcome of patients treated for cervical myelopathy. A prospective, multicenter study with independent clinical review. Spine. 2000;25:670-6.

6. Casha S, Engelbrecht HA, DuPlessis SJ, Hurlbert RJ. Suspended laminoplasty for wide posterior cervical decompression and intradural access: results, advantages, and complications. J Neurosurg Spine. 2004;1:80-6.

7. Edwards CC II, Heller JG, Murakami H. Corpectomy versus laminoplasty for multilevel cervical myelopathy: an independent matched-cohort analysis. Spine (Phila Pa 1976). 2002;27:1168-75.

8. Tetreault L, Ibrahim A, Côté P Singh A, Fehlings MG. A systematic review of clinical and surgical predictors of complications following surgery for degenerative cervical myelopathy. J Neurosurg Spine. 2016;24:77-99.

9. Emery SE. Cervical spondylotic myelopathy: diagnosis and treatment. J Am Acad Orthop Surg. 2001;9:376-88.

10. Bakhsheshian J, Mehta VA, Liu JC. Current diagnosis and management of cervical spondylotic myelopathy. Global Spine J. 2017;7:572-86.

11. Baron EM, Young WF. Cervical spondylotic myelopathy: a brief review of its pathophysiology, clinical course, and diagnosis. Neurosurgery. 2007;60:S35-41.

12. Iyer A, Azad TD, Tharin S. Cervical spondylotic myelopathy. Clin Spine Surg. 2016;29:408-14.

13. Nurick $S$. The pathogenesis of the spinal cord disorderassociated with cervical spondylosis. Brain. 1972;95:87-100.

14. Donnally CJ, Butler AJ, Rush AJ, Bondar KJ, Wang MY, Eismont FJ. The most influential publications in cervical myelopathy. J Spine Surg. 2018;4:770-9.

15. Albayrak HK, Kazanci A, Gurcay AG, Gurcan O, Ozates MO. Clinical and radiological comparison of surgical treatment methods in patients with cervical spinal stenosis. J Turk Spinal Surg. 2020;31:88-95.

16. McCormick JR, Sama AJ, Schiller NC, Butler AJ, Donnally CJ. Cervical spondylotic myelopathy: a guide to diagnosis and management. Am Board Fam Med. 2020;33:303-13.

17. Donnally IC, Munakomi S, Varacallo M. Basilar invagination. Treasure Island, FL: StatPearls; 2018.

18. Fehlings MG, Tetreault LA, Riew KD, Middleton W, Wang JC. A clinical practice guideline for the management of degenerative cervical myelopathy: introduction, rationale, and scope. Global Spine J. 2017;7(3 Suppl): 21S-7S.

19. Lawrence BD, Shamji MF, Traynelis VC, Yoon ST, Rhee JM, Chapman JR, et al. Surgical management of degenerative cervical myelopathy: a consensus statement. Spine. 2013;38:S171-2.

20. Fehlings MG, Wilson JR, Kopjar B, Yoon ST, Arnold PM, Massicotte EM, et al. Efficacy and safety of surgical decompression in patients with cervical spondylotic myelopathy: results of the AOSpine North America prospective multi-center study. I Bone Joint Surg Am. 2013;95:1651-8. 